\title{
The Design of the Context Quantizer on the Basis of Amazing Measure
}

\author{
YiPing Zhang ${ }^{1,}$ a , Min Chen ${ }^{2, b}$ * \\ ${ }^{1}$ Department of Science, Yunnan Police Office Academy, China \\ ${ }^{2}$ Information Security College, Yunnan Police Office Academy, China \\ Corresponding Author: Min Chen forestcm@163.com
}

\begin{abstract}
Keywords: Context Modeling; Context Quantization; Amazing Measure; Clustering Algorithm
\end{abstract}
\begin{abstract}
When using the clustering algorithm, the implementation of the context quantization not only expands the application range of the quantizer, but also obtains better coding performance. However, those clustering algorithms depend on the choice of the similarity measure. In the the previous works, the increment of the description length was suggested but the result is that it cannot fully meets the similarity measure of various attributes, resulting in the performance of the clustering result deviation. In this paper, a new similarity measure which holds better mathematical description is given. The increment of the amazing measure, which denotes the similarity measure, two count vectors are discussed in this paper and its corresponding properties are also explained. The experimental results indicate that when using the proposed similarity measure, both the stability of the context quantizer and the corresponding coding results can be optimized at the same time.
\end{abstract}

\section{Introduction}

Efficient context modeling entropy coding technique [1] always plays an important role in the field of digital signal compression. Modeling is the key to enhancing the compression efficiency, which takes advantage of the correlation between source symbols as much as possible. By conditioning for the current symbol, the entropy of the conditional probability distribution is reduced. Actually, in practice, these distributions are estimated through the counting approach and then used to drive the arithmetic encoder for assigning the code word for each symbol. Theoretically, the entropy of the conditional probability distribution is the lower band of the code length for coding every symbol. It means that if the entropy is low, coding compression effect is more ideal. However, there are a variety of factors influencing the coding performance. One important of them is the context dilution. In training or coding process, each count vector obtains its corresponding data and the number of these data is used to estimate distributions [2]. If the number of data is not enough to achieve the distribution with high efficiency, the distribution will be trend to the uniform distribution which holds the highest entropy. The context quantization is an efficient approach to tackle the context dilution. Actually, the context dilution makes the coding result including high model cost [3]. In order to make each count vector containing more number of data, in tegrating some count vectors into one is an ideal method. It is also the way what context quantization does. The first context quantizer is CALIC algorithm in [4], which uses the empirical quantizer to partition the space of the context. After modifying, CALIC could achieve ideal coding results. The strict optimized context quantizers are the minimum conditional entropy context quantization (MCECQ) in [5,6] and the minimum adaptive code length context quantization (MCLCQ) in [7,8]. However, these two quantizers have their flaws. MCECQ relies on the input of the quantization levels, which implies that MCECQ cannot achieve the optimized class adaptively. MCLCQ cannot obtain the optimized results for I-ary source only for binary case. Actually, in $[9,10]$, the context quantization based on the clustering algorithms are suggested. In [9], the modified genetic algorithm is used to cluster the context model for achieving the quantization levels ad aptively. In [10], the increment of the description length is defined as the similarity measure between two conditional probability distributions and the affinity propagation is employed to implement the context quantization. Both of these two quantizers can achieve the similar results with the previous 
optimized context quantizers. However, the increment of the description length is not the strictly similarity measure, which limit the performance of the coding system.

Actually, in the derivation of the increment of the description length, there are new items to be considered. In this paper, researchers call it the amazing measure. Meanwhile, the increment of the amazing measure is also discussed in the the present work, including the features which indicate that the increment of the amazing measure is suit for judging whether two conditional probability distributions are similar. Then the increment of the amazing measure is used for the context quantization to improve the coding efficiency.

\section{Amazing Measure}

In the context modeling entropy coding, the conditional probability distributions are unknown. Usually, these distributions are estimated by using their corresponding count vectors. When a count vector $\mathbf{V}=\left\{n_{0}, n_{1}, \ldots, n_{I-1}\right\}$ is given, the estimator is described by (1)

$$
p(x=i)=\frac{n_{i}+\delta}{\sum_{i=0}^{I-1} n_{i}+I \delta}
$$

Where ${ }^{n_{i}}$ denotes the number of symbols whose value is ${ }^{i}$ and $\delta$ denotes the initial number of the count vector. When each probability is calculated by (1), the conditional probability distribution is determined. Its' corresponding description length under the given count vector can be calculated by (2)

$$
L=\log (n+I-1) !-\sum_{i=1}^{I} \log n_{i} !-\log (I-1) !
$$

Where ${ }^{n}$ denotes the total nu mber of symbols the count vector contains. Mean while, according to [x], the representation (2) can also be formed as (3)

$$
L=n * H(x \mid C)+\Delta
$$

Where ${ }^{H(x \mid C)}$ denotes the entropy of the conditional probability distribution, which are from the estimation by using the count vector $\mathbf{v}$. $\Delta$ denotes the model cost to describe the context model. In a lot of previous researches, the objective of context quantizers is to minimize the description length $L$. Actually, from (4), it is obviously that if $H(x \mid C)$ is small, the total description length $L$ will not be large. With this discussion, the context quantization can aim to minimize the entropy of the estimated distribution. However, the entropy is unknown so that the objective of the optimization is lost. However, actually, along with the estimation process, when more and more symbols are coded, they are used to obtain new estimation. The conditional probability distribution on the current time is different from those in different times, which implies that the current entropy is the value that can be calculated. On the other hand, the uniform distribution holds the maximu m value of entropy. It means that if values of ${ }^{n_{i}}$ in the count vector $\mathbf{v}$ are similar with each other. The resulted distribution will tend to the uniform distribution. It is opposite to the objective. Based on this purpose, it is necessary to determine the state of the count vector at the current time weather this count vector is trending to the uniform distribution. In this paper, researchers give the definition of the amazing measure to denote the state above. For the count vector $\mathbf{V}$, its current amazing measure is described by (4)

$$
\xi=\log \frac{\prod_{i=0}^{I-1} n_{i}}{\sum_{i=0}^{I-1} n_{i}}=\log \frac{\prod_{i=0}^{I-1} n_{i}}{n}
$$

From(4), it is easy to find that if each value of ${ }^{n_{i}}$ is larger than 1 , and the value of ${ }^{\zeta}$ is larger than 0 . In the coding process, it means that the count vector obtain the counts of symbols, which implies that if a count vector is efficient, the corresponding amazing measure can holds a no-negative value. Mean while, in [x], the increment of the description length $\Delta L$ is represented by (5) 


$$
\Delta L=N_{1} * D\left(p\left(x \mid c_{1}\right) \| p\left(x \mid c_{m k}\right)\right)+N_{2} * D\left(p\left(x \mid c_{2}\right) \| p\left(x \mid c_{m k}\right)\right)-\frac{I-1}{2} \log \frac{N_{1} N_{2}}{N_{1}+N_{2}}
$$

In this representation, $\Delta L$ contains the amazing measure. Researchers gave the discussion that the increment of the description length was equivalent to the similarity measure between two count vectors (actually two conditional probability distributions estimated by count vectors respectively). The first form of (5) can be considered as the weighting of the relative entropy. The relative entropy is the similarity measure of two distributions. Then if this equality will be satisfied, the second form of (5) is needed to holds the properties that the similarity measure have. It indicates that the amazing measure can also be used as the similarity measure.

When clustering algorithm is suggested to implement the context quantization, the similarity measure should be determined firstly. In practice, the purpose with this similarity measure is to choose the two count vector to merge under the criterion that they are similar. Actually, if two same count vectors are merged, the amazing measure of the merged count vector will not be changed. Namely, the zero value of the amazing measure can be seemed as the criterion that two count vectors are the same one. Then researchers can obtain the representation (6) to express the similarity of two count vectors:

$$
\Delta \zeta=\left(\zeta_{1}+\zeta_{2}\right)-\zeta_{1,2}
$$

Where ${ }^{\Delta \zeta}$ denotes the increment of the amazing measure when two count vectors whose amazing measures are $\zeta_{1}$ and $\zeta_{2}$ respectively. Meanwhile, it is easy to find that $\Delta \zeta \geq 0$. Then researchers use $\Delta \zeta$ as the similarity measure to implement the context quantization.

\section{Context Quantization based on the Minimum Increment of the Amazing Measure}

When the similarity measure is determined, many clustering algorithms, such as K-means and Affinity propagation, can be used to implement the context quantization. The steps of the context quantization based on the clustering algorithm with the similarity measure, amazing measure, are listed as follows:

Step 1: When determining the order of the context model, initializing the context model.

Step 2: Updating the count vectors with the process of the coding.

Step 3: If the number of symbols encoded comes to the given number, go to step4, otherwise, go to step 2 for coding.

Step 4: Calculating the increment of the amazing measure by (4) and (6). Then the chosen clustering algorithm is employed to implement the context quantization. When the quantization process is end, go back to Step 2 to continue coding.

\section{Experiments and Results}

In the experiments, the proposed context quantizer is employed to compress the image sources. Similar with the reference [2], K-means is used to implement the context quantization. The only difference is the amazing measure is used in the experiments instead of the Euclid distance used in [8]. For simplify ing experiment, 6 images are quantized into 8 levels and the 4 of themare used to train the context model with order $3,{ }^{P\left(x \mid x_{1}, x_{2}, x_{3}\right)}$. The last 2 images (lena, barb) are used as the coding source.

After quantization and coding, the number of clusters and the code length (bit) are listed in Table 1. Table 1 The Comparison of the Coding Results by Two Context Quantizer

\begin{tabular}{ccccc}
\hline \multirow{2}{*}{ image } & \multicolumn{2}{c}{ proposed } & \multicolumn{2}{c}{ KMCQ } \\
\cline { 2 - 5 } & cluster & Code length (bit) & cluster & Code length (bit) \\
\hline barb & 231 & 256901 & 213 & 259260 \\
\hline lena & 231 & 174325 & 192 & 179252 \\
\hline
\end{tabular}


From Table 1, it is obvious that the code length from the proposed algorithm is better than the result from KMCQ in [8]. The reason is that the amazing measure is related to both the count vector and the entropy. Namely, both the space correlation and statistic correlation are contained in the amazing measure. Based on this similarity measure, the clustering algorithm can achieve the better results which are ben efit by the clustering result more reasonable. Mean while, the context quantization based on the amazing measure can obtain stable quantization level, which the KMCQ cannot remain.

Above all, the increment of the amazing measure is more suitable for the similarity measure. The objective of the context quantization is achieved.

\section{Conclusions}

In this paper, a new similarity measure which holds better mathematical description is given. The increment of the amazing measure, which denotes the similarity measure two count vectors are discussed in this paper and its corresponding properties are also explained. The experimental results indicate that when using the proposed similarity measure, both the stability of the context quantizer and the corresponding coding results can be optimized at the same time.

\section{Acknowledgement}

This work was supported by Natural Science Foundation of Yunnan under Grant 2013FD042.

\section{References}

[1] J. Rissanen, A universal data compression system, IEEE Trans. Inform. Theory, 1983, 29: 656 664.

[2] J. Rissanen and G. Langdon, "Universal modeling and coding," IEEE Trans. Inf. Theory, 1981, 27(1): $12-23$.

[3] X. Wu, G. Zhai, Adaptive Sequential Prediction of Multidimensional Signals with Applications to Lossless Image Coding, IEEE Trans. Image Processing, 2011, 20(1):36-42.

[4] X. Wu, Lossless Compression of Continuous-tone Images via Context Selection and Quantization, IEEE Trans. on Image Proc, 1996, 6(5):656 - 664.

[5] Jianhua Chen, Yufeng Zhang, Xinling Shi, Image Coding based on Wavelet Transform and Uniform Scalar Dead Zone Quantizer, Signal Processing: Image Communication, 2006, 21: 562-572.

[6] Jianhua Chen, Context Modeling based on Context Quantization with Application in Wavelet Image Coding, IEEE Trans. Image Processing, 2004, 13(1):26-32.

[7] X. Wu. Context Quantization with Fisher Discriminant for Adaptive Embedded Wavelet Image Coding, Proc. of 1999 Data Compression Conference, pp. 102-111, Mar. 1999.

[8] Min Chen, Jianhua Chen, Context Quantization based on the Modified Genetic Algorithm with K-mean s, Proceeding of 9th Intemational Conference on Natural Co mputation, 424-428, Shengy ang China, 2013.7.

[9] Min Chen, Jianhua Chen, Affinity Propagation for the Context Quantization, Advanced Materials Research, 2013, 791:1533-1536. 